\title{
Metagenomic approach to study biofilm in medical context
}

\section{Author:}

Suzana Meira Ribeiro ${ }^{1}$

Alinne Pereira de Castro ${ }^{1}$

Octávio Luiz Franco ${ }^{\mathbf{1 , 2}}$

\section{Affiliations:}

1 S-Inova Biotech, Programa de Pos-Graduação em Biotecnologia, Universidade Catolica Dom Bosco, Campo Grande, MS, Brazil

2 Centro de Analises Proteomicas e Bioquimicas, Pos-Graduação em Ciencias Genomicas e Biotecnologia, Univrsidade Catolica de Brasilia, Brasilia, DF, Brazil

\section{Correspondence author:}

Octávio Luiz Franco

E-mail: ocfranco@gmail.com

\begin{abstract}
Microbial organization in biofilms plays an important role in health and disease. This microbial lifestyle can protect its host against pathogens and contribute to the metabolism of nutrients. On the other hand, pathogenic biofilms can increase the morbidity and mortality of patients, raising the economic cost of infections. Here, we describe how biofilm is organized, its role in different scenarios in the human body and discuss how metagenomic studies has contributed to understand the microbial diversity that interact with the host. It is currently known that many biofilms in the human body may have a polymicrobial composition. In the context of infectious diseases, this composition may limit therapeutic approaches to combat biofilms even more. Thus, describing microbial diversity could help to guide therapeutic decisions.
\end{abstract}

Keywords: Biofilm, metagenomic and polymicrobial infection 


\section{Introduction}

Infectious diseases play a pivotal role in medicine system by causing worldwide health problems. Emerging infectious disease have become prevalent, causing illness, death and huge healthcare costs (1). The major challenges are infectious diseases caused by antibiotic-resistant bacteria or infectious diseases caused by novel pathogens. Microbial resistance limits the antibiotics action reducing its potential to combat infections. Furthermore, it threatens the advances in medicine such as surgeries - a practice that may expose patients to infections, including those caused by the resistant microorganisms.

Currently, it is known that infectious diseases may be caused not only by one single potential pathogenic microorganism but instead by a personalized combination of complex interactions of distinct microbial species (2). Such a combination can be organized as biofilm (microorganisms encased in extracellular matrix) (Figure 1). This microbial lifestyle confers to cells within the biofilm protection against environmental stress, such as desiccation and antibiotic action.

Biofilms formed by different bacteria species or even a mix of bacteria and fungi, present advantages when compared to biofilms formed by a single microbial specie. Interspecific relationship may result in metabolic cooperation (consequently, more advantage to sustain the microbial life within the biofilm) and higher resistance to environmental stress. In biofilm, microbial cells susceptible to antibiotics, for example, can benefit from protection displayed by other cells resistant to antibiotics. In this scenario of passive resistance, studies showed that a $\beta$-lactamase-producing strain of non-typeable Haemophilus influenzae (NTHi 86-028NP) could protect Staphylococcus pneumoniae in vivo through $\beta$-lactamase production (3).

Nowadays, our understanding is still uncertain about the complex microbial diversity involved during health and disease situations. In general, studies have demonstrated that metagenomic prospections as a possible tool for clinical diagnosis. Besides this, metagenomic studies can demonstrate the distribution of microbes in space and time, allowing a better understanding of the ecological implications involved in the microbial relationship. This could guide therapies to balance the indigenous human microbiome or to combat pathogenic biofilms.

\section{Biofilms in medical scenario}

In the last thirty years, studies have shown that most microorganisms live as biofilm instead of planktonic lifestyle (4). Currently, it is known that most of microbial life on the planet exists as polymicrobial biofilm consortium. Cells in the biofilm are encased by a matrix that consists of extracellular polymeric substances, that may include polysaccharides, nucleic acids, lipids and proteins (5). The biofilm matrix can interact with the environment promoting its adhesion to surfaces. 


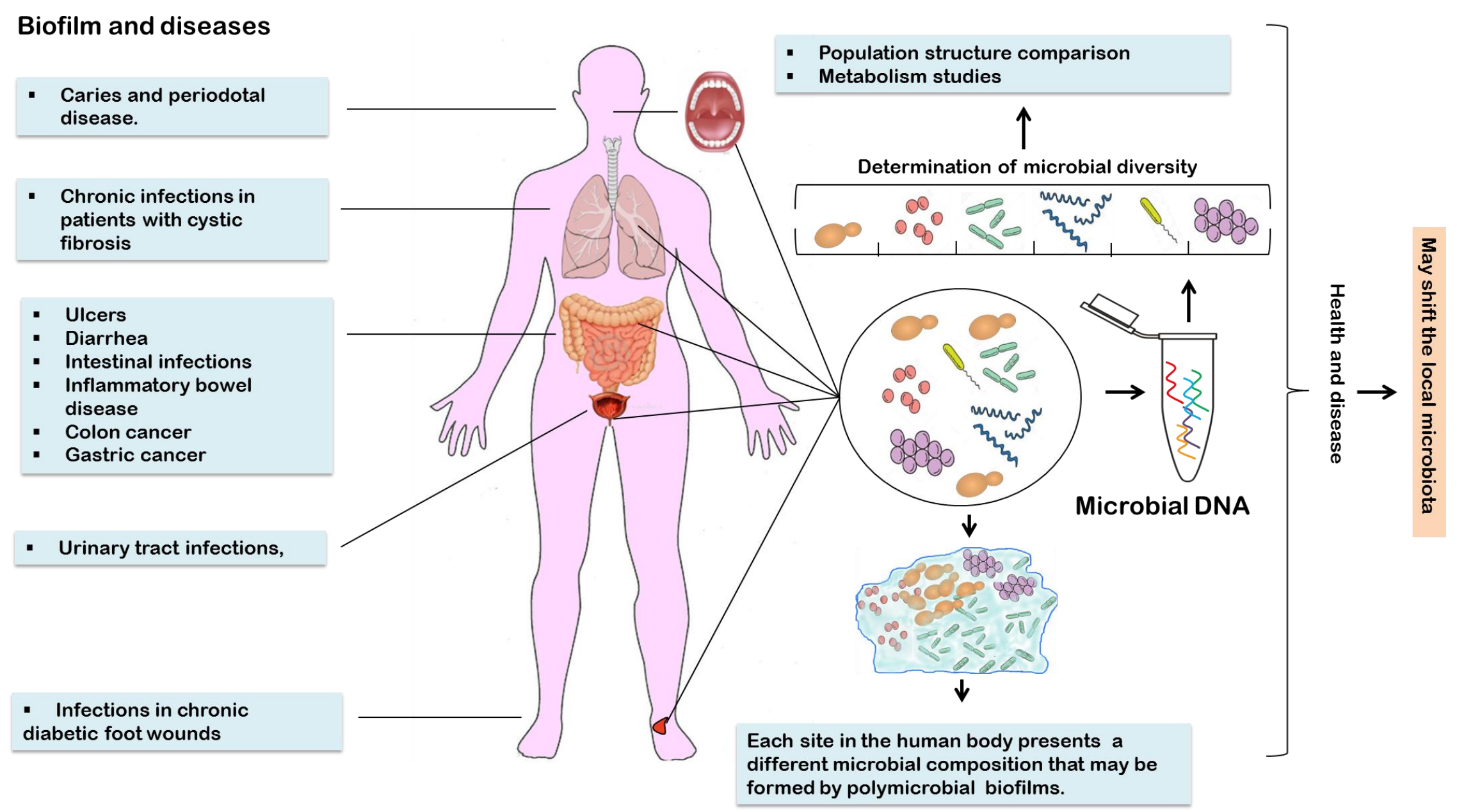

Figure 1. Diseases associated with biofilms and the metagenomic approach to determine microbial diversity in different sites of the human body 


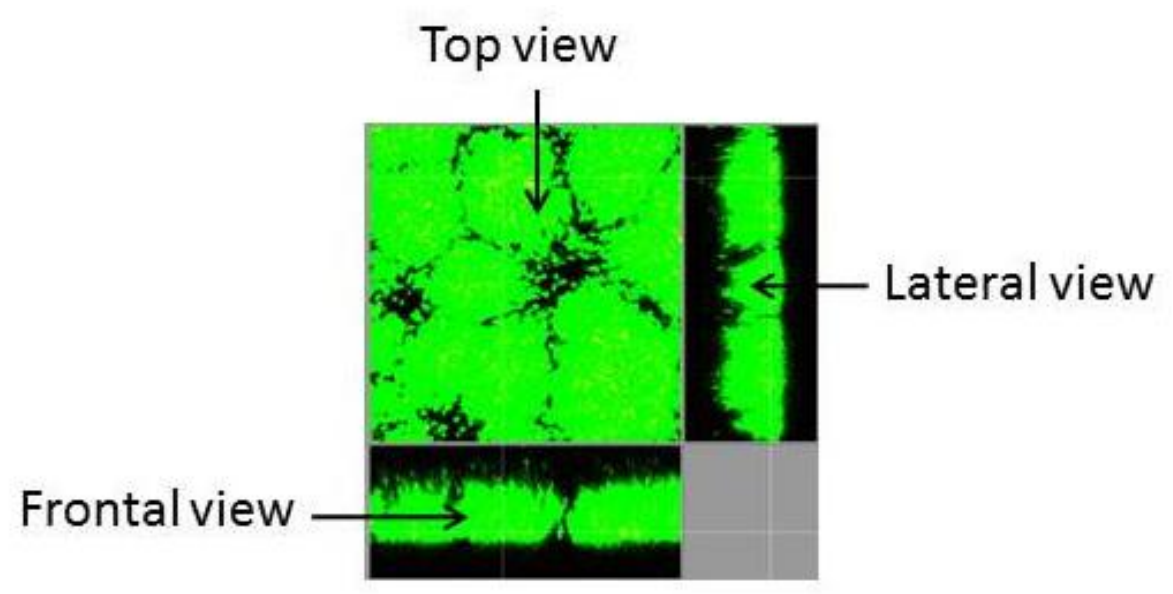

Figure 2. Three-dimensional structure of Klebsiella pneumonia ATCC 13883 biofilm. The image shows the bacteria stained with Syto-9 stain and analyzed using confocal microscopy (unpublished data).

Microorganisms which live in the biofilm community can adhere to each other, forming flocks, which are mobile biofilms not fixed to substratum or a layer that adhere to surfaces (Figure 2) (5). When biofilms develop and become mature, cells from them can detach, colonize other surfaces and form other biofilms. In the biofilm "house", microbial cells can get nutrients while they are protected by environmental stresses. In medical context, such an environmental stress can be host defence, antibiotics and products used to clean medical devices or environments. In addition, life in close proximity favours the exchange of genetic material by conjugation, increasing the gene pool (6). The polymicrobial composition of biofilms and the ability to realize lateral transference of genes may potentiate the biofilm resistance to environmental stresses $(6,7)$.

Biofilms can display two sides of a same coin, a "good side", associated to beneficial aspects to their host including protection against pathogenic microorganism or even contributing for a better immune defence. Otherwise a "bad side" was also observed, associated with infections and even to other diseases such as cancer (Figure 1) $(8,9)$.
Microbial biofilms that establish symbiotic mutualistic relationship with humans can be found in different sites of the body surfaces such as the skin and intestinal tract $(10,11)$. Indigenous bacteria that colonize the skin such as Staphylococcus epidermids has the ability to form biofilm (12). This bacteria can protect the skin against colonization and infections caused by $S$. aureus (13). The intestines present the largest and complex human body microbiota (14). This microbiota may interact with human host cells and further contribute to metabolism and biosynthesis of compounds necessary for biological processes in the human body (15).

Disruptions in a biofilm that is part of an indigenous microbiome in the human body can compromise the microbial homeostasis, predisposing the host to diseases (16). Antibiotics, for example, can disrupt the biofilm that inhabits the human intestinal tract, affecting the host protection against pathogens and limiting the availability of nutrients and vitamin production (17). Imbalance in intestinal microbiota (dysbiose) can also contribute to the development of colorectal cancer (CRC) (18). In this cancer type, opportunistic pathogens form polymicrobial biofilm on local mucosa, contributing to CRC progression. Studies 
have shown that there exists a metabolic association between biofilm and CRC (22).

The oral scenario is a space for microbiota diversity and its composition has been associated to the health and diseases in humans. Loss of microbiota has been linked to periodontitis progression, an inflammatory disease that affect tissues that support the teeth (19). The local inflammation is caused by pathogenic bacteria that destroy the periodontal connective tissue, potentially leading to teeth loss. Studies have indicated that the core microorganisms that persist in sub gingival biofilms could represent pathogens associated with establishment of disease $(19,20)$. As observed in several contexts of the human body, changes in normal microbiota can affect the health of their host. A possibility to minimize diseases associated with the imbalance of microbiota could consist of restoring the original microbiota.

In clinical environments, biofilms are considered problematic, since they can be a source of infection. They can directly colonize human surfaces or they colonize medical devices and contaminate patients that depend of such devices as catheters, endotracheal tubes, joint implants, breast implants and others (21-24). The biofilm formed in these devices serves as a source of pathogenic microorganisms that may cause acute or chronic infections. In some contexts, medical devices can facilitate the spread of microbiota present in a specific part of the human body to other locations, causing infections. Endotracheal tubes for example, can favour the spread of oral microbiota present in dental plaque to lungs and could cause ventilator-associated pneumonia (23).

Infections caused by biofilms are normally difficult to treat due to the inherent resistance of this lifestyle to current antibiotics (8). In addition, single cells that form biofilm may present genes that confer resistance to antibiotic. In this scenario, even if a biofilm is disrupted, the antibiotic resistant free cells can survive. Once free, these cells can colonize new environments, forming biofilms or can spread through the body causing systemic or local infections. This context is particularly a worldwide worrying problem, since most antibiotics are ineffective against resistant microorganisms and biofilm lifestyle (25).

\section{Metagenomic: application in clinical context}

Sometimes, in the infectious diseases there is not only one single potential pathogenic microorganism responsible to make the disorder but instead, a personalized combination of complex interactions of distinct microbial species are expected (2). Currently, we are still uncertain in our understanding of this complex microbial diversity involved during health and disease situations. In general, studies have demonstrates the prospect of metagenomics as a possible tool for clinical diagnosis. Surveillance and discovery efforts have been focused in studies of normal physiology as well as in the investigation of acute diseases like diarrhea, hemorrhagic fevers, pneumonia, among others classic infectious diseases (26-29) .

The following example provides evidence of the potential for metagenomics approach for the identification of microbial pathogens during unhealthy situations being relevant to the field of modern medical microbiology. Ultimately, based on traditional culture-dependent approach, it has long been believed that the lung was sterile, therefore the metagenomics microbial identification data set has revealed the true microbial diversity associated with lungs (30). In 2011, metagenomics has been used in a clinical diagnostic setting to identify the cause of outbreak of diarrheal disease (28). In this regard, a Shiga-toxigenic Escherichia coli (STEC) outbreak occurred in the Germany, with more than 3000 cases and 50 deaths 
(31). In this case, metagenomic analyses were able to recover the identification of bacterial pathogens during this outbreak by genome sequences where initial efforts to culture the bacteria infectious agent failed. Another comparison involves the extracted genomic information from six patient samples collected from the same hospital within a period of a few weeks (32). During this analysis, nearly 400 wholegenome sequencing (WGS) libraries from 124 clinical bacterial isolates of $P$. aeruginosa were constructed. More intriguing was the authors' suggestion that the microbial signature is consistent with community-acquired infection or infection of these patients from their own microbiota in the hospital setting. It is now evident the relevance of the use of sequence-based metagenomics to resolve cases involving unclear infectious diseases.

Over the last two decades, metagenomics in combination with nextgeneration-sequencing technology, has enabled us to improve our understanding of the discovery of new potential pathogens, the rapid resolution into clinical diagnostics, antibiotic resistance detection and insights into infection control (33-35). Providing a powerful methodological approach to accelerate our current knowledge on how microorganisms could affect our health.

Although metagenomics is considered a great promising strategy for microbial diagnostics, this rapidly emerging area of study presents some challenges. An important consideration is that all these progresses rely on the development of sequencing technologies. Thus, extensive efforts have been carried out to rely on sophisticated computational tools, and particular interests are for the importance of skilled bioinformatics support for the implementation and use of metagenomics in the clinic. As a consequence, these massive datasets will soon become a part of the routine toolkit in clinical diagnostic laboratories with profound implications for the medical management within the healthcare system.

\section{Metagenomic as strategy to study biofilms}

Interactions among the microbial diversity in the biofilm are complex and play a pivotal role in the pathogenesis of infections. Studies using well-established metagenomic analyses have demonstrated a shift from monomicrobial to polymicrobial biofilm studies in infectious diseases (36) (Figure 1). It has previously been reported that microorganisms that possess a biofilm strategy have mechanisms for recruiting other microorganisms as partners, mainly by quorum sensing molecules, improving their survivability - consequently, it can cause polymicrobial infection (37).

Given the considerable massive number of people that annually develop chronic infections, there has been significant interest for a detailed understanding of polymicrobial infection. In large part, this is due to the microbial diversity availability, followed by the interactions between synergistic genes in the polymicrobial biofilm - it can increase the severity of the infection. In principle, the biofilms associated with the lung, ear, oral and urinary tract are the most common polymicrobial biofilms studied. For example, high bacterial diversity was detected in the sputum of cystic fibrosis patients with a substantial decline in lung function. The authors showed that this microbial diversity was composed of Pseudomonas aeruginosa, Rothia mucilaginosa, Streptococcus pneumoniae and Candida albicans instead of only Streptococcus pneumonia singlespecies biofilm (38).

In addition, efforts have been done to understand the periodontal disease and caries, since they are the most prevalent infectious diseases in humans. Dental plaque is also a polymicrobial biofilm. In 
this context, using metagenomic analysis, Liu and colleagues (39) have allowed for an increasingly detailed study to compare the metabolism between healthy and periodontal disease samples. This wellestablished analysis showed that periodontal disease samples were enriched with genes associated with fatty acid metabolism, acetyl-CoA degradation and energy-coupling factor class transporters when compared with healthy samples. Otherwise, the authors emphasized the enrichment of genes involved in the homoserine metabolism (a signaling molecule for quorum sensing) in healthy samples. As discussed earlier, this similar quorum sensing molecule can suggest a metabolic activity within the healthy microbiome oral biofilm.

Another study has demonstrated the ability of the reproducible mixed-species biofilm (Pseudomonas aeruginosa, Pseudomonas protegens and Klebsiella pneumoniae) to be more resilient to antimicrobials than single-species biofilms. Intriguingly, the increase of mixed-species biofilm resistance was detected as an unknown protection mechanisms offered by resistant bacteria to all members in the mixed-species biofilm (40).

Finally, synergy between polymicrobial biofilms has been described in patients with infected diabetic foot ulcers (DFUs) as well (41). Diabetic foot infections represent a significant chronic disease and molecular analysis brought additional insights into the bacterial diversity that may be responsible for DFU chronicity. In addition to others studies, Mottola and collaborators (2016) showed that DFU polymicrobial biofilms are composed of Staphylococcus, Corynebacterium, Enterococcus, Pseudomonas and Acinetobacter genera. All these examples cited above reveal great progress in our understanding in which polymicrobial biofilm contribute to the pathogenesis of several infectious disorders. For this reason, molecular diagnostics makes metagenomic studies an attractive strategy in both research and diagnostics. Additionally, direct sequencing of metagenomic DNA, can be performed to reveal the genetic exchange, interspecies interactions and signaling within harmful biofilms (42).

\section{Conclusions}

Besides access to the microbial noncultivated diversity, metagenomic studies have extended our observation that biofilms can be formed by numerous species, including members of different kingdoms. Such polymicrobial organization may affect the action of antimicrobials and antibiofilms agents, limiting the therapeutic approaches. In this context, metagenomic studies can contribute to clarify our understanding of microbial diversity and about its interactions with distinct biotic and abiotic factors in human. The continuous advances in metagenomic investigations can provide new biotechnological potentials for the diagnosis of infection and for application in therapies. 


\section{REFERENCES}

1. Carlet J, Collignon P, Goldmann D, Goossens H, Gyssens IC, Harbarth S, et al. Society's failure to protect a precious resource: antibiotics. The Lancet. 2011 Jul 23;378(9788):36971.

2. Bertelli C, Greub G. Rapid bacterial genome sequencing: methods and applications in clinical microbiology. Clin Microbiol Infect Off Publ Eur Soc Clin Microbiol Infect Dis. 2013 Sep;19(9):803-13.

3. Weimer KED, Juneau RA, Murrah $\mathrm{KA}$, Pang B, Armbruster CE, Richardson $\mathrm{SH}$, et al. Divergent mechanisms for passive pneumococcal resistance to $\beta$-lactam antibiotics in the presence of Haemophilus influenzae. J Infect Dis. 2011 Feb 15;203(4):549-55.

4. Battin TJ, Besemer $\mathrm{K}$, Bengtsson MM, Romani AM, Packmann AI. The ecology and biogeochemistry of stream biofilms. Nat Rev Microbiol. 2016 Apr;14(4):251-63.

5. Flemming H-C, Wingender J, Szewzyk U, Steinberg P, Rice SA, Kjelleberg S. Biofilms: an emergent form of bacterial life. Nat Rev Microbiol. 2016 Sep;14(9):563-75.

6. Cook LCC, Dunny GM. The influence of biofilms in the biology of plasmids. Microbiol Spectr. 2014 Oct 10;2(5):12.

7. Kean R, Rajendran R, Haggarty J, Townsend EM, Short B, Burgess KE, et al. Candida albicans Mycofilms Support Staphylococcus aureus Colonization and Enhances Miconazole Resistance in DualSpecies Interactions. Front Microbiol. 2017;8:258.
8. Hughes G, Webber MA. Novel approaches to the treatment of bacterial biofilm infections. $\mathrm{Br} \mathrm{J}$ Pharmacol. 2017 Jan 1;n/a-n/a.

9. Johnson $\mathrm{CH}$, Dejea CM, Edler D, Hoang LT, Santidrian AF, Felding $\mathrm{BH}$, et al. Metabolism Links Bacterial Biofilms and Colon Carcinogenesis. Cell Metab. 2015 Jun 2;21(6):891-7.

10. Macfarlane S, Bahrami B, Macfarlane GT. Mucosal biofilm communities in the human intestinal tract. Adv Appl Microbiol. 2011;75:111-43.

11. Percival SL, Emanuel C, Cutting KF, Williams DW. Microbiology of the skin and the role of biofilms in infection. Int Wound J. 2012 Feb;9(1):14-32.

12. Büttner H, Mack D, Rohde H. Structural basis of Staphylococcus epidermidis biofilm formation: mechanisms and molecular interactions. Front Cell Infect Microbiol. 2015;5:14.

13. Iwase T, Uehara Y, Shinji H, Tajima A, Seo H, Takada K, et al. Staphylococcus epidermidis Esp inhibits Staphylococcus aureus biofilm formation and nasal colonization. Nature. 2010 May 20;465(7296):346-9.

14. Vos WM de. Microbial biofilms and the human intestinal microbiome. $\mathrm{Npj}$ Biofilms Microbiomes. 2015 Mar 25;1:15005.

15. Hattori M, Taylor TD. The Human Intestinal Microbiome: A New Frontier of Human Biology. DNA Res Int J Rapid Publ Rep Genes Genomes. 2009 Feb;16(1):1-12.

16. Marsh PD, Zaura E. Dental biofilm: ecological interactions in health and 
disease. J Clin Periodontol. 2017 Mar 1;44:S12-22.

17. Guarner F, Malagelada J-R. Gut flora in health and disease. Lancet Lond Engl. 2003 Feb 8;361(9356):512-9.

18. Li S, Konstantinov SR, Smits R, Peppelenbosch MP. Bacterial Biofilms in Colorectal Cancer Initiation and Progression. Trends Mol Med. 2017 Jan;23(1):18-30.

19. Ai D, Huang R, Wen J, Li C, Zhu J, Xia LC. Integrated metagenomic data analysis demonstrates that a loss of diversity in oral microbiota is associated with periodontitis. BMC Genomics. 2017;18(1):1041.

20. Curtis MA, Zenobia C, Darveau RP. The relationship of the oral microbiotia to periodontal health and disease. Cell Host Microbe. 2011 Oct 20;10(4):302-6.

21. Gbejuade HO, Lovering AM, Webb JC. The role of microbial biofilms in prosthetic joint infections. Acta Orthop. 2015 Mar 4;86(2):147-58.

22. Rieger UM, Mesina J, Kalbermatten DF, Haug M, Frey HP, Pico R, et al. Bacterial biofilms and capsular contracture in patients with breast implants. Br J Surg. 2013 May 1;100(6):768-74.

23. Sands KM, Wilson MJ, Lewis MAO, Wise MP, Palmer N, Hayes AJ, et al. Respiratory pathogen colonization of dental plaque, the lower airways, and endotracheal tube biofilms during mechanical ventilation. J Crit Care. 2017 Feb;37:30-7.

24. Stickler DJ. Clinical complications of urinary catheters caused by crystalline biofilms: something needs to be done. J Intern Med. 2014 Aug 1;276(2):120-9.
25. Rather IA, Kim B-C, Bajpai VK, Park Y-H. Self-medication and antibiotic resistance: Crisis, current challenges, and prevention. Saudi J Biol Sci [Internet]. [cited 2017 Feb 20]; Available from: http://www.sciencedirect.com/science /article/pii/S1319562X17300049

26. Dunne WM, Westblade LF, Ford B. Next-generation and whole-genome sequencing in the diagnostic clinical microbiology laboratory. Eur J Clin Microbiol Infect Dis Off Publ Eur Soc Clin Microbiol. 2012 Aug;31(8):1719-26.

27. Greninger AL, Chen EC, Sittler T, Scheinerman A, Roubinian N, Yu G, et al. A metagenomic analysis of pandemic influenza A (2009 H1N1) infection in patients from North America. PloS One. 2010 Oct 18;5(10):e13381.

28. Loman NJ, Constantinidou C, Christner M, Rohde H, Chan JZ-M, Quick J, et al. A culture-independent sequence-based metagenomics approach to the investigation of an outbreak of Shiga-toxigenic Escherichia coli O104:H4. JAMA. 2013 Apr 10;309(14):1502-10.

29. Padmanabhan R, Mishra AK, Raoult D, Fournier P-E. Genomics and metagenomics in medical microbiology. J Microbiol Methods. 2013 Dec;95(3):415-24.

30. Dickson RP, Erb-Downward JR, Huffnagle GB. Towards an ecology of the lung: new conceptual models of pulmonary microbiology and pneumonia pathogenesis. Lancet Respir Med. 2014 Mar;2(3):238-46.

31. Frank C, Werber D, Cramer JP, Askar M, Faber M, an der Heiden M, et al. Epidemic profile of Shiga-toxinproducing Escherichia coli O104:H4 
outbreak in Germany. N Engl J Med. 2011 Nov 10;365(19):1771-80.

32. Kim S, Jonghe JD, Kulesa $\mathrm{AB}$, Feldman D, Vatanen T, Bhattacharyya RP, et al. Highthroughput automated microfluidic sample preparation for accurate microbial genomics. Nat Commun. 2017 Jan 27;8:13919.

33. Fischer $\mathrm{N}$, Rohde $\mathrm{H}$, Indenbirken $\mathrm{D}$, Günther T, Reumann K, Lütgehetmann $\mathrm{M}$, et al. Rapid metagenomic diagnostics for suspected outbreak of severe pneumonia. Emerg Infect Dis. 2014 Jun;20(6):1072-5.

34. Lewandowska DW, Zagordi O, Zbinden A, Schuurmans MM, Schreiber P, Geissberger F-D, et al. Unbiased metagenomic sequencing complements specific routine diagnostic methods and increases chances to detect rare viral strains. Diagn Microbiol Infect Dis. 2015 Oct;83(2):133-8.

35. Loman NJ, Pallen MJ. Twenty years of bacterial genome sequencing. Nat Rev Microbiol. 2015;13(12):787-94.

36. Gabrilska RA, Rumbaugh KP. Biofilm models of polymicrobial infection. Future Microbiol. 2015;10(12):1997-2015.

37. Wolcott R, Costerton JW, Raoult D, Cutler SJ. The polymicrobial nature of biofilm infection. Clin Microbiol Infect Off Publ Eur Soc Clin Microbiol Infect Dis. 2013 Feb;19(2):107-12.

38. Paganin P, Fiscarelli EV, Tuccio V, Chiancianesi M, Bacci G, Morelli P, et al. Changes in cystic fibrosis airway microbial community associated with a severe decline in lung function. PloS One. 2015;10(4):e0124348.

39. Liu B, Faller LL, Klitgord N, Mazumdar V, Ghodsi M, Sommer DD, et al. Deep sequencing of the oral microbiome reveals signatures of periodontal disease. PloS One. 2012;7(6):e37919.

40. Lee KWK, Periasamy S, Mukherjee M, Xie C, Kjelleberg S, Rice SA. Biofilm development and enhanced stress resistance of a model, mixedspecies community biofilm. ISME J. 2014 Apr;8(4):894-907.

41. Mottola C, Mendes JJ, Cristino JM, Cavaco-Silva P, Tavares L, Oliveira M. Polymicrobial biofilms by diabetic foot clinical isolates. Folia Microbiol (Praha). 2016 Jan;61(1):35-43.

42. McLean RJC, Kakirde KS. Enhancing metagenomics investigations of microbial interactions with biofilm technology. Int J Mol Sci. 2013 Nov 11;14(11):22246-57. 